\title{
Extending Adjacency to Fuzzy Sets for Coping with Imprecise Image Objects
}

\author{
Isabelle Bloch, Henri Maître \\ École Nationale Supérieure des Télécommunications \\ Département Images - CNRS URA 820 \\ 46 rue Barrault, 75013 Paris, France \\ Tel: +3314581 75 85, Fax: +3314581 37 94, E-mail: bloch@ima.enst.fr
}

\section{Introduction}

Adjacency has a large interest in image processing and pattern recognition, since it qualifies an important relationship between image objects or regions. A crisp definition of adjacency between crisp objects often leads to a low robustness in case of noise or segmentation errors. Let us consider for instance a problem of model-based pattern recognition where spatial relationships are an important part of the recognition process. If two model objects are adjacent, we expect the corresponding image objects to be adjacent too, otherwise they will be difficult to recognize. However, if classical crisp adjacency is used, the fact that two objects are adjacent or not may depend on one point only.

In order to include possible errors or imprecision in the processing and in the recognition, we use the framework of fuzzy sets that already proved to be useful for image processing under imprecision. Two ways can be considered for representing imprecision. In the first one, the satisfaction of the adjacency property between two objects is considered to be a matter of degree. The second one consists in introducing imprecision in the objects themselves, and to deal with fuzzy objects, i.e. with objects considered as fuzzy sets on the image space. For instance, spatial imprecision due to the limited quality of image information can be represented in an adequate way by considering fuzzy objects. Then obviously adjacency is also a matter of degree. In both cases, a need exists to give proper definitions for fuzzy adjacency between image regions. Here the second way only will be considered (see [3] for an approach along the first track).

Unfortunately, only a few works address the problem of fuzzy adjacency in the literature. Fuzzy topology was introduced in [7]. In this paper, Rosenfeld defines a fuzzy connectivity between points but without reference to fuzzy neighborhood, or to fuzzy adjacency. Similar approaches can be found in [8], [11], where degrees of connectivity in a fuzzy set are also introduced, but neither the connectivity nor the adjacency between two fuzzy sets are defined. Rosenfeld and Klette [9] define a degree of adjacency between two crisp or fuzzy sets, using a geometrical approach based on the notion of "visibility" of a set from another one. However, this definition is not symmetrical, and probably not easy to extend to higher dimensions. We propose in this paper a completely different approach. The closest to our work is probably the one decribed in [4], where a degree of adjacency between two fuzzy sets is defined by extending binary definitions of contours, frontiers, and neighborhood. Again, the proposed definition of [4] is not symmetrical, and presents some other drawbacks.

In this paper we propose several definitions for degree of adjacency coping with spatial imprecision in image processing. Basic definitions for classical no- 
tions of adjacency are given in Section 2, in the discrete domain. Then we account for imprecision in the representation of objects, consequently defined as spatial fuzzy sets, and we define a degree of adjacency between two fuzzy sets. This is detailed in Section 3, using fuzzy extensions of the notions of neighborhood and of boundary of a set. We finally show that fuzzy mathematical morphology provides a consistent framework for expressing the obtained definitions.

\section{Crisp adjacency}

Here, we restrict ourselves to the discrete case, and use discrete topology as derived from discrete connectivity for defining adjacency between two image regions $X$ and $Y$, subsets of the discrete space. Consider an $n$-dimensional discrete space (typically $\mathbb{Z}^{n}$ ), and any discrete connectivity defined on this space, denoted cconnectivity (for instance, for $n=3$, we may consider 6-, 18- or 26-connectivity on a cubic grid). Since we would like to distinguish between connectedness and adjacency relationships, we use the following definition of crisp adjacency, where we denote by $n_{c}(x, y)$ the Boolean variable stating that $x$ and $y$ are neighbors in the sense of the discrete c-connectivity:

Definition 1 For any two subsets $X$ and $Y$ in $\mathbb{Z}^{n}, X$ and $Y$ are adjacent according to the c-connectivity if: $X \cap Y=\emptyset$ and $\exists x \in X, \exists y \in Y: n_{c}(x, y)$.

We consider for the discrete boundary of a set $X$ its interior boundary defined as: $\partial X=X-E\left(X, B_{c}\right)$ where $E\left(X, B_{c}\right)$ denotes the morphological erosion of $X$ by the structuring element $B_{c}$ of size 1 defined according to the chosen discrete connectivity [10]. Using this definition, the discrete adjacency can be related to the boundary in the following way:

Property 1 A consequence of definition 1 is that, if $X$ and $Y$ are adjacent, then any $x \in X$ and $y \in Y$ that satisfy $n_{c}(x, y)$ belong to the boundary of $X$ and $Y$ respectively.

Therefore the fuzzy extension of definition 1 can be obtained either by considering only the constraint on the neighborhood, or by considering also the constraint on the boundary, as will be seen in Section 3.2.

Property 2 Definition 1 can also be expressed equivalently in terms of morphological dilation, as: $X \cap Y=\emptyset$ and $D\left(X, B_{c}\right) \cap Y \neq \emptyset, \quad D\left(Y, B_{c}\right) \cap X \neq \emptyset$, where $D\left(X, B_{c}\right)$ denotes the dilation of $X$ by the structuring element $B_{c}$.

This property provides a third way to extend the definition to fuzzy sets, either directly from fuzzy dilation, or by means of distance computation, which is closely related to dilation.

\section{Extending adjacency to fuzzy objects}

In the rest of this text we consider fuzzy objects (i.e. fuzzy sets defined on the considered space by means of their membership function) and define fuzzy adjacency between such objects. In the discrete case, a fuzzy object is simply defined by its membership function, defined on $\mathbb{Z}^{n}$ and taking values in $[0,1]$.

\subsection{Methods}

This Section presents shortly the possible principles that can be used for extending adjacency to fuzzy sets and the requirements posed to this extension. We 
consider the general problem of extending a relationship $R_{B}$ between two binary objects to its fuzzy equivalent $R$ (fuzzy relationship between two fuzzy objects). Instantiations of the described methods to the case of adjacency are provided next.

By using the $\alpha$-cuts One way to define crisp sets from a fuzzy set consists in taking the $\alpha$-cuts of this set. Therefore a first class of methods relies on the application of the relationship $R_{B}$ to each $\alpha$-cut. This gives rise to two different "fuzzification" methods in the literature.

The first one consists in "stacking" the results obtained with binary operations on the $\alpha$-cuts: let us denote by $\mu$ and $\nu$ the membership functions of two fuzzy objects defined on the considered space and taking values in $[0,1]$, the fuzzy equivalent $R$ of $R_{B}$ is then defined as (see e.g. [5]): $R(\mu, \nu)=\int_{0}^{1} R_{B}\left(\mu_{\alpha}, \nu_{\alpha}\right) d \alpha$, or similarly by a double integration (other fuzzification equations exist, but will not be examined here). Examples of this approach concern for instance connectivity [8], fuzzy mathematical morphology [2], distances [5], [1], etc.

The second method is the extension principle [12], which leads, in the general case, to a fuzzy number: $\forall n \in \mathcal{V}\left(R_{B}\right), R(\mu, \nu)(n)=\sup _{R_{B}\left(\mu_{\alpha}, \nu_{\alpha}\right)=n} \alpha$, where $\mathcal{V}\left(R_{B}\right)$ denotes the image of $R_{B}$, i.e. the set of values taken by $R_{B}$. If the relationship to be extended only takes binary values $(0 / 1$, or true/false), then the extension principle reduces to: $R(\mu, \nu)=\sup _{R_{B}\left(\mu_{\alpha}, \nu_{\alpha}\right)=1} \alpha$. This is typically the case for binary adjacency between binary sets.

By formal translation of equations $A$ second class of methods consists in translating binary equations into their fuzzy equivalent: intersection is replaced by a t-norm, union by a t-conorm, sets by membership functions, etc. This has been used e.g. for defining fuzzy morphology [2]. This translation is straightforward if the binary relationship can be expressed in set theoretical and logical terms. It is obtained in a natural way from the definitions given in Section 2. Moreover, this remark endows methods based on mathematical morphology with a particular interest, since mathematical morphology is mainly based on set theory.

Since methods based on $\alpha$-cuts appeared to have poorer properties than those based on a formal translation (see [3]), we restrict to this second kind of approach in the following.

Minimal properties required for a fuzzy adjacency The properties we require for fuzzy adjacency are the following: symmetry, consistency with binary definitions, decreasingness with respect to the distance between both sets. A last property, often desirable although not mandatory, is invariance with respect to geometrical transformations.

\subsection{Formal translation of binary adjacency equations into fuzzy adjacency}

In this Section, we make use of the principle shortly described in Section 3.1 in order to define a degree of adjacency between two fuzzy sets $\mu$ and $\nu$. Since binary definitions always involve constraints on the intersection of the two sets 
and a notion of neighborhood, we first define fuzzy equivalents of these concepts. Then, we extend definition 1, using only neighborhood relationships, and then, we add boundary constraints, as introduced in property 1 . We also consider fuzzy adjacency derived from fuzzy dilation and from fuzzy distance.

Degree of intersection between two fuzzy sets The degree of intersection between two fuzzy sets is obtained by translating the set equation $X \cap Y \neq \emptyset$ into fuzzy terms. This equation is equivalent to $\exists x \in \mathbb{Z}^{n}, x \in X \cap Y$. The simplest fuzzy translation provides: $\mu_{i n t}(\mu, \nu)=\sup _{x} t[\mu(x), \nu(x)]$, where $t$ is any t-norm [6]. The supremum is taken over the whole space. A degree of empty intersection (or of disjunctness) is then derived as: $\mu_{\neg i n t}(\mu, \nu)=c\left[\mu_{i n t}(\mu, \nu)\right]$, where $c$ is a fuzzy complementation (for instance defined as $\forall a \in[0,1], c(a)=1-a$ ). This form is not always adequate for image processing purposes since it does not incorporate any spatial information. Degrees of intersection and of nonintersection can therefore be reformulated in order to better reflect the spatial overlapping by considering the fuzzy hypervolume of the intersection. This may also be interpreted as a translation process, in the sense that we have: $X \cap Y=$ $\emptyset \Leftrightarrow V_{n}(X \cap Y)=0$. For defining the hypervolume of a fuzzy set, we simply use the classical fuzzy cardinality. This provides for a fuzzy set $\mu$ (with a bounded support) in the discrete case: $V_{n}(\mu)=\sum_{x \in \mathbb{Z}^{n}} \mu(x)$. From the hypervolume of $t(\mu, \nu)$, a degree of intersection in $[0,1]$ is derived. It should be equal to 0 if $\mu$ and $\nu$ have completely disjoint supports, be high if one set is included in the other, and increasing with respect to the hypervolume of the intersection. The following definition satisfies these requirements:

Definition 2 The degree of intersection between two fuzzy sets $\mu$ and $\nu$, depending on the hypervolume of their intersection, is defined by:

$\mu_{i n t}(\mu, \nu)=\frac{V_{n}[t(\mu, \nu)]}{\min \left[V_{n}(\mu), V_{n}(\nu)\right]}$. Here again a degree of non-intersection can be derived from this expression using fuzzy complementation.

Property 3 The intersection degrees defined by maximum of inlersection and definition 2 are both consistent with the binary definition and invariant with respect to geometrical transformations.

In the following definitions of fuzzy adjacency, we may use either expressions derived from the height of the intersection or expressions involving the fuzzy hypervolume of the intersection. We will see that this leads to different adjacency degrees in overlapping situations.

Fuzzy neighborhood In this Section, we define a degree of neighborhood $n_{x y}$ between two points $x$ and $y$ in $\mathbb{Z}^{n}$ endowed with a discrete connectivity. Let us first consider binary definitions of $n_{x y}$ : we set $n_{x y}=1$ if $x$ and $y$ are neighbors in the sense of the considered discrete connectivity, and $n_{x y}=0$ otherwise (i.e. $\left.n_{x y}=n_{c}(x, y)\right)$. With this definition, the consistency with the binary case is guaranteed. In the fuzzy case, $n_{x y}$ can be defined as a decreasing function of the distance between $x$ and $y$, as proposed in [4] and in [3].

Using neighborhood constraints We propose to fuzzify definition 1 by combining a degree of empty intersection $(X \cap Y=\emptyset)$ with a degree of existence of neighbors $\left(\exists x \in X, \exists y \in Y, n_{c}(x, y)\right)$ using a t-norm $t$ (expressing the simultaneous satisfaction of both conditions). For the first part, a degree of nonintersection can be used, derived either from the height of the intersection of 
from its fuzzy hypervolume, as suggested in Section 3.2. For the second part, existence is translated by means of a supremum (taken over the whole space), leading to: $\sup _{x} \sup _{y} t\left[\mu(x), \nu(y), n_{x y}\right]$, where $n_{x y}$ represents the degree to which $x$ and $y$ are neighbors ${ }^{1}$. It can be either crisp or fuzzy (as defined in Section 3.2). Finally, we obtain the following definition for fuzzy adjacency.

Definition 3 The degree of adjacency between $\mu$ and $\nu$ involving only neighborhood constraints is defined as: $\mu_{a d j}(\mu, \nu)=t\left[\mu_{\neg i n t}(\mu, \nu), \sup _{x} \sup _{y} t\left[\mu(x), \nu(y), n_{x y}\right]\right]$.

Property 4 The degree of adjacency obtained with this definition is symmetrical, consistent with the discrete binary definition (i.e. in the case where $\mu$ and $\nu$ are crisp and $n_{x y}=n_{c}(x, y)$ ), and decreasing with respect to the distance between the two fuzzy sets. It is invariant with respect to geometrical transformations (for scaling, only if $n_{x y}$ is itself invariant).

Figure 1 illustrates the results obtained with definition 3 with the t-norm minimum and both definitions of degree of intersection. Using the maximum of the intersection we obtain $\mu_{a d j}(\mu, \nu)=0.36$ and $\mu_{a d j}\left(\mu, \nu^{\prime}\right)=0.35$, which are very similar values. On the contrary, using the fuzzy hypervolume, definition 3 accounts for the differences in intersection and provides $\mu_{\text {adj }}(\mu, \nu)=0.67$ and $\mu_{a d j}\left(\mu, \nu^{\prime}\right)=0.34$, which are different and better fit the intuition.

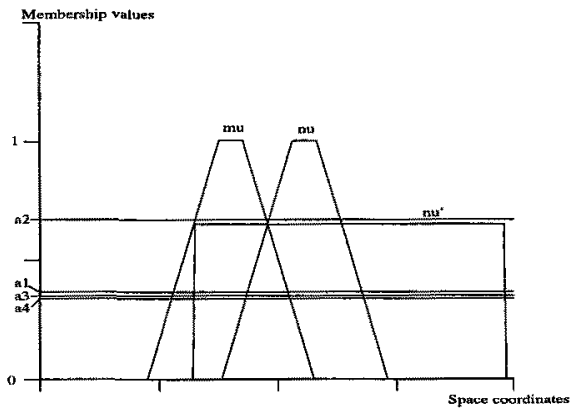

Fig. 1. Illustration of definition 3 when using different definitions for the degree of intersection. Using the maximum of intersection we obtain $\mu_{a d j}(\mu, \nu)=a_{1}(=0.36)$ and $\mu_{a d j}\left(\mu, \nu^{\prime}\right)=a_{3}(=0.35)$, and using the fuzzy hypervolume $\mu_{a d j}(\mu, \nu)=a_{2}(=0.67)$ and $\mu_{a d j}\left(\mu, \nu^{\prime}\right)=a_{4}(=0.34)$.

Let us consider now a 2D example. Figure 2 shows a slice of a magnetic resonance (MR) image of the human brain, where several structures have been segmented and serve as a model (or atlas), and a slice (at approximately the same level) of another MR image where the same structures have to be recognized. A rough fuzzy segmentation of this second image is also shown. The adjacency degrees between some of the obtained fuzzy objects are given in Table 1 . They are obtained using definition 3 with the maximum of intersection as intersection degree and the t-norm minimum. 4-connectivity was used. The results are in agreement with what can be expected from the model. In this case, crisp adjacency would provide completely different results in the model and in the image, preventing its use for recognition. This suggests that fuzzy adjacency degree can indeed be used for pattern recognition purposes, of course combined with other spatial relationships.

Adding boundary constraints Another way to extend fuzzy adjacency from definition 1 consists in introducing a constraint on the boundary of the considered

${ }^{1}$ In such expressions $t(a, b, c)$ stands for $t[t(a, b), c]$. This notation is adopted for sake of simplicity and justified since any $t$-norm is commutative and associative. 

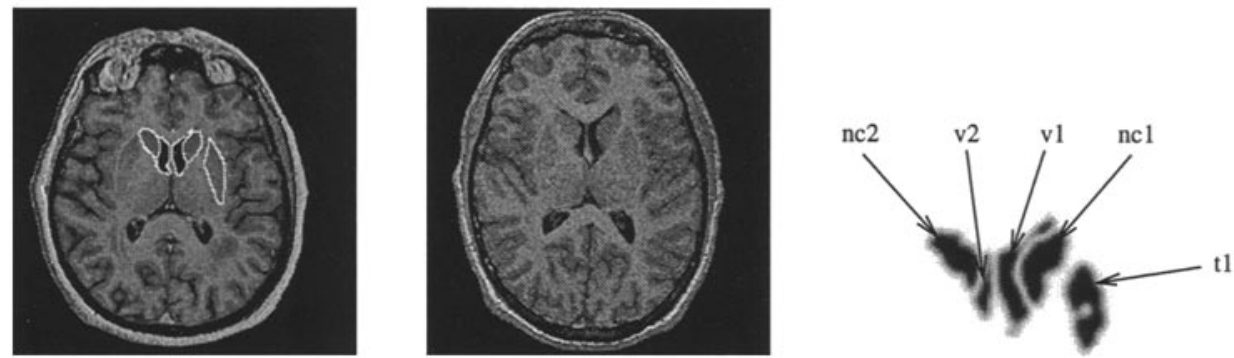

Fig. 2. MR image of a brain with a few segmented structures (left). MR image of another brain (middle). Right: 5 fuzzy objects resulting from a rough fuzzy segmentation of the middle image (membership values rank between 0 and 1 , from white to black, the maximum membership value is displayed at each point) and labels (used in Table 1).

\begin{tabular}{|c|c|c|c|}
\hline Fuzzy object 1 & Fuzzy object 2 & degree of adjacency & adjacency in the model (crisp) \\
\hline v1 & $\mathrm{v} 2$ & 0.368 & 1 \\
v1 & nc1 & 0.463 & 1 \\
v1 & $\mathrm{t} 1$ & 0.000 & 0 \\
v1 & $\mathrm{nc2}$ & 0.035 & 0 \\
v2 & $\mathrm{nc2}$ & 0.427 & 1 \\
nc1 & $\mathrm{t} 1$ & 0.035 & 0 \\
\hline
\end{tabular}

Table 1. Results obtained using definition 3 with the maximum of intersection as intersection degree, the t-norm minimum, and 4-connectivity. Labels of structures are given in Figure 2. High degrees are obtained between structures where adjacency is expected, while very low degrees are obtained in the opposite case.

sets, as given by property 1 , i.e. the neighbor points involved in definition 1 are on the boundary of the sets.

A similar work has already be done in [4], but although the approach is very attractive, the proposed definitions suffer from several drawbacks, with respect to the requirements we imposed in this paper (not symmetrical, not consistent with the binary case, etc., see [3] for more details).

We propose a new definition that overcomes these drawbacks and better matches our requirements. Our approach consists in defining only the fuzzy boundaries of the fuzzy sets, which are then combined with neighborhood relationship.

Definition 4 The fuzzy boundary of a fuzzy set $\mu$ is defined by the membership function $b_{\mu}$ as: $\forall x \in \mathbb{Z}^{n}, b_{\mu}(x)=t\left[\mu(x), \sup _{z \in \mathbb{Z}^{n}} t\left[c(\mu)(z), n_{x z}\right]\right]$.

Property 5 In the binary case ( $\mu$ and $n_{x z}$ binary), this definition is consistent with the classical definition of the boundary of a crisp set $X$ (set of points of $X$ that have a neighbor in $X^{C}$ ). It is also invariant with respect to geometrical transformations (for scaling, only if $n_{x y}$ is itself invariant).

The translation of definition 1 along with the property on boundary leads now to the following definition:

Definition 5 The degree of adjacency between $\mu$ and $\nu$ involving neighborhood and boundary constraints is defined by:

$\mu_{\text {adj }}(\mu, \nu)=t\left[\mu_{\neg \text { int }}(\mu, \nu), \sup _{x} \sup _{y} t\left[b_{\mu}(x), b_{\nu}(y), n_{x y}\right]\right]$, where the supremum is taken over $\mathbb{Z}^{n}$.

This definition is illustrated in Figure 3. 


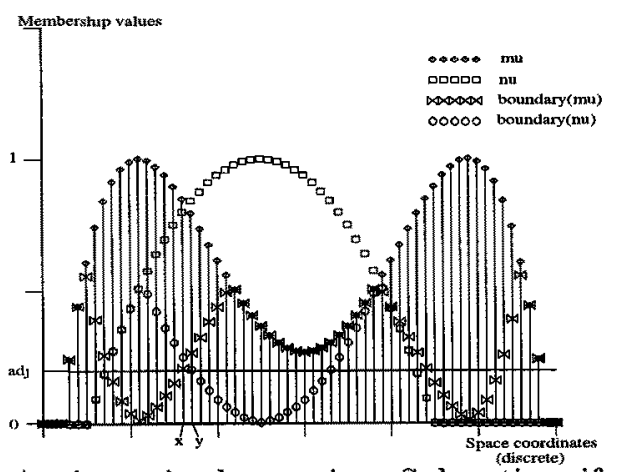

Fig. 3. Fuzzy boundary $b_{\mu}$ and $b_{\nu}$ of two fuzzy sets $\mu$ and $\nu$. Fuzzy adjacency is then equal to adj according to definition 5 , which is less than $a_{2}$ provided by definition 3 . The neighbor points for which the adjacency value is attained are again $x$ and $y$.

Again, as in the previous Subsection, if we assume that the considered fuzzy sets constitute a fuzzy partition, we can ignore the first term corresponding to the degree of empty intersection.

Property 6 The degree of adjacency in this definition is symmetrical, consistent with the binary definition if $\mu, \nu$ and $n_{x y}$ are binary, and decreases if the distance between $\mu$ and $\nu$ increases. It is invariant with respect to geometrical transformations (for scaling, only if $n_{x y}$ is itself invariant).

Property 7 This definition is equivalent to definition 3 in the binary case. In the fuzzy case, it is more severe, i.e. leads to a lower degree of adjacency.

Using fuzzy morphological operators In a morphological context, it may also be interesting to define adjacency from fuzzy dilation, by translating property 2 into fuzzy terms. A direct translation of this property leads to the following definition.

Definition 6 The degree of adjacency between $\mu$ and $\nu$ involving fuzzy dilation is defined as: $\mu_{a d j}(\mu, \nu)=t\left[\mu_{\neg i n t}(\mu, \nu), \mu_{i n t}\left[D\left(\mu, B_{c}\right), \nu\right], \mu_{i n t}\left[D\left(\nu, B_{c}\right), \mu\right]\right]$. This definition represents a conjunctive combination of a degree of non-intersection between $\mu$ and $\nu$ and a degree of intersection between one fuzzy set and the dilation of the other. $B_{c}$ can be taken as the elementary structuring element related to the considered connectivity, or as a fuzzy structuring element, representing for instance spatial imprecision (i.e. the possibility distribution of the location of each point).

Property 8 This definition is symmetrical, consistent with the binary definition if $\mu, \nu$ and $B_{c}$ are binary, and decreases if the distance between $\mu$ and $\nu$ increases.

Fuzzy dilation can also serve for defining the fuzzy boundary of a fuzzy set, as follows.

Definition 7 The fuzzy boundary $b_{\mu}$ of a fuzzy set $\mu$ is defined from fuzzy dilation as: $b_{\mu}(x)=t\left[\mu(x), D\left(c(\mu), B_{c}\right)(x)\right]$.

Property 9 This definition is equivalent to definition 4 if the structuring element is consistent with the choice of the fuzzy neighborhood (typically if we take the elementary structuring element defined from the discrete connectivity used in a binary definition of $n_{x y}$ ).

Property 10 The degree of adjacency obtained from this boundary definition (with any structuring element) is still symmetrical, consistent with the binary case and decreasing when the distance between both fuzzy sets increases. 
Finally, since in the discrete binary case the equation using dilation means that the minimum (nearest point) distance between $X$ and $Y$ is equal to 1 , we can also exploit this fact in the fuzzy case, by using the fuzzy minimum distance, defined from fuzzy dilation as in [1]. We do not go into further details for this approach, since it leads to similar definitions, sharing the same properties as the previous ones.

\section{Conclusion}

The aim of this research was to investigate notions of fuzzy adjacency that could serve for model-based pattern recognition in image processing under imprecision. We proposed several definitions for extending adjacency to fuzzy objects, that show good properties with respect to binary definitions and to the requirements we imposed, and that provide for a consistent representation and management of imprecision, which is directly represented in the considered objects.

Acknowledgment: This work has been partially supported by the "Conférence des Grandes Écoles" (we would like to thank in particular Prof. Michel Perrin) and has been initiated while the first author was visiting at BISC group, Computer Science Department, University of California at Berkeley (Profs. Zadeh and Anvari).

\section{References}

1. I. Bloch. Distances in Fuzzy Sets for Image Processing derived from Fuzzy Mathematical Morphology. In Information Processing and Management of Uncertainty in Knowledge-Based Systems, volume III, pages 1307-1312, Granada, Spain, July 1996.

2. I. Bloch and H. Maitre. Fuzzy Mathematical Morphologies: A Comparative Study. Pattern Recognition, 28(9):1341-1387, 1995.

3. 1. Bloch, H. Maître, and M. Anvari. Fuzzy Adjacency between Image Objects. International Journal of Uncertainty, Fuzziness and Knowledge-Based Systems, 5(6), 1997.

4. C. Demko and E. H. Zahzah. Image Understanding using Fuzzy Isomorphism of Fuzzy Structures. In IEEE Int. Conf. on Fuzzy Systems, pages 1665-1672, Yokohama, Japan, March 1995.

5. D. Dubois and M.-C. Jaulent. A General Approach to Parameter Evaluation in Fuzzy Digital Pictures. Pattern Recognition Letters, 6:251-259, 1987.

6. D. Dubois and H. Prade. Combination of Information in the Framework of Possibility Theory. In M. Al Abidi et al., editor, Data Fusion in Robotics and Machine Intelligence. Academic Press, 1992.

7. A. Rosenfeld. Fuzzy Digital Topology. Information and Control, 40:76-87, 1979.

8. A. Rosenfeld. The Fuzzy Geometry of Image Subsets. Paltern Recognition Letters, $2: 311-317,1984$.

9. A. Rosenfeld and R. Klette. Degree of Adjacency or Surroundness. Pattern Recognition, 18(2):169-177, 1985.

10. J. Serra. Image Analysis and Mathematical Morphology. Academic Press, London, 1982.

11. J. K. Udupa and S. Samarasekera. Fuzzy Connectedness and Object Definition: Theory, Algorithms, and Applications in Image Segmentation. Graphical Models and Image Processing, 58(3):246-261, 1996.

12. L. A. Zadeh. The Concept of a Linguistic Variable and its Application to Approximate Reasoning. Information Sciences, 8:199-249, 1975. 